\title{
A Morse COMPlex on manifolds With Boundary
}

\author{
FRANÇOIS LAUDENBACH
}

\begin{abstract}
Given a compact smooth manifold $M$ with non-empty boundary and a Morse function, a pseudo-gradient Morse-Smale vector field adapted to the boundary allows one to build a Morse complex whose homology is isomorphic to the (absolute or relative to the boundary) homology of $M$ with integer coefficients. Our approach simplifies other methods which have been discussed in more specific geometric settings.
\end{abstract}

\section{INTRODUCTION}

We consider a smooth compact manifold $M$ of dimension $n$ with a non-empty boundary $\partial M$. A smooth function is said to be Morse when its critical points lie in the interior of $M$, are not degenerate, and when its resriction to the boundary is Morse. The property of being Morse in this sense, with distinct critical values, is generic among the smooth functions on $M$. Let $f: M \rightarrow \mathbb{R}$ be a Morse function. There are two types of critical points of $f \mid \partial M$, called type $N$ and D (we shall see later that N (resp. D) is like Neumann (resp. Dirichlet)): a critical point $p \in \partial M$ is of type $N$ (resp. D) when $<d f(p), n(p)>$ is negative (resp. positive), where $n(p)$ denotes the outward normal to the boundary at $p$. Looking at the change of homotopy type of the sub-level set $M^{a}:=\{x \in M \mid f(x) \leq a\}$ when $a$ is increasing, it is well known that change happens when $a$ crosses a critical value of $f \mid \operatorname{int}(M)$ or of $f \mid \partial M$ only when they are of type $N$; no change happens when crossing a critical value of type $D$.

Thirty years ago, I tried to find Morse inequalities in this setting. Of course, $f$ gives rise to a Morse function $D f$ on the double manifold $D M:=M \underset{\partial M}{\cup} M$. But Morse inequalities for $D f$ are not sharp. At that time I did not succeed in finding a geometrical Morse complex in the case of manifolds with boundary. I had even kept in mind the idea that such a complex should not exist. Apparently, the problem seems to be still open, at least in the setting of generic assumptions on the boundary (see below).

In a seminal paper [20, E. Witten introduced a deformed Laplacian (now called the Witten Laplacian) on a closed Riemannian manifold equipped with a Morse function and deduced in particular an analytic proof of the Morse inequalities. Recently, Francis Nier explained to me various works concerning the case of manifolds with non-empty boundary. K. Chang and J. Liu [5] introduced two Witten complexes of differential forms constrained to satisfy some boundary conditions. Two types of boundary conditions can be distinguished: Dirichlet conditions which cancel tangential components or Neumann conditions which cancel normal components. These

2000 Mathematics Subject Classification. 57R19.

Key words and phrases. Morse theory, pseudo-gradient.

supported by the French ANR program 'Floer Power". 
authors made the analysis easier by considering only flat metrics near the critical points. These boundary problems with general metrics have been recently studied in [10] (Helffer-Nier) for the Dirichlet problem, in [11] (Kolan-Prokhorenkov-Shubin) for Dirichlet and Neumann, and in [13] (Le Peutrec) for the Neumann problem, with additional developments concerned with the asymptotic analysis.

In case of the Dirichlet conditions [5] [10], when the deformation parameter $h$ is small enough, the De Rham complex $\Omega^{*}$ contains a finite dimensional sub-complex $F^{*}$ (of $\mathbb{R}$-vector spaces) whose cohomology (when $M$ is orientable) is isomorphic to the relative cohomology $H^{*}(M, \partial M ; \mathbb{R})$. In that case, a basis of $F^{*}$ is in a bijective correspondence with the critical points of $f$ in $\operatorname{int}(M)$ and those of type $D$ on the boundary (up to a shift of their grading). In case of the Neumann boundary conditions [5] [13], a similar result holds: $F^{*}$ is generated by the critical points of $f$ in the interior and by those of type $N$ on the boundary; the cohomology of $F^{*}$ is isomorphic to the singular cohomology of $M$ with real coefficients. Hence, Morse inequalities follow. Let us also mention work by M. Braverman and V. Silantyev ([2]) which is in the same spirit and deals with the Morse-Novikov theory. But they introduce some extra condition which obliges them to exclude for instance the standard annulus in the plane equipped with the height function.

There are also approaches motivated by Floer homology. In [1], M. Akaho considers a very specific geometric situation along the boundary. In [9], P. Kronheimer and T. Mrowka consider functions and their gradients having extensions to the double manifold $D M$ which are invariant by the canonical symmetry. Both settings are non-generic, and the setup and analysis are complicated by the presence of isolated trajectories in the boundary which preserves Morse index.

These results (mainly coming from analysis) beg the existence of a simpler geometric Morse complex associated to any Morse function on a manifold with boundary. In this note, we are going to perform this program, working with $\mathbb{Z}$-coefficients (or orientation-twisted coefficients) rather than with real cohomology as in Witten's work. We use the following notation:

- $C_{k}$ denotes the set of critical points of $f: \operatorname{int}(M) \rightarrow \mathbb{R}$ of index $k$;

- $N_{k}$ denotes the set of critical points of $f: \partial M \rightarrow \mathbb{R}$ of type $N$ and index $k$;

- $D_{k}$ denotes the set of critical points of $f: \partial M \rightarrow \mathbb{R}$ of type $D$ and index $k-1$ (notice that such a point has index $k$ in the double manifold $D M$ ).

$-|\cdot|$ stands for the cardinality of the mentioned finite set.

Theorem A. Let $F_{*}^{N}$ be the free graded $\mathbb{Z}$-module generated by $C_{*} \cup N_{*}$. There exists a differential $\partial: F_{*}^{N} \rightarrow F_{*-1}^{N}$, making $\left(F_{*}^{N}, \partial\right)$ a chain complex, such that the homology of $\left(F_{*}^{N}, \partial\right)$ is isomorphic to the singular homology $H_{*}(M, \mathbb{Z})$.

As usual, Morse inequalities follow (see J. Milnor [15], p. 28, or R. Bott [3], p. 338). They are contained in a polynomial identity. Let $\mathcal{M}_{f}^{N}(T)$ be the Morse polynomial of type $N$, where $T$ is the variable:

$$
\mathcal{M}_{f}^{N}(T)=\sum_{k}\left|C_{k} \cup N_{k}\right| T^{k}
$$


Let $\mathcal{P}_{M}(T)$ be the Poincaré polynomial of $M$

$$
\mathcal{P}_{M}(T)=\sum_{k} \operatorname{rank} H_{k}(M ; \mathbb{Z}) T^{k}
$$

Corollary A. We have $\mathcal{M}_{f}^{N}(T)-\mathcal{P}_{M}(T)=(1+T) Q^{N}(T)$, where $Q^{N}(T)$ is a polynomial with non-negative coefficients.

Making now the critical points of type $D$ play the main role yields the following statement.

Theorem B. Let $F_{*}^{D}$ be the graded $\mathbb{Z}$-module generated by $C_{*} \cup D_{*}$. There exists a co-differential $d: F_{*}^{D} \rightarrow F_{*+1}^{D}$, making $\left(F_{*}^{D}, d\right)$ a cochain complex, such that the cohomology of $\left(F_{*}^{D}, d\right)$ is isomorphic to the relative cohomology $H^{*}\left(M, \partial M ; \mathbb{Z}^{\text {or }}\right)$ with coefficients twisted by the orientation of $M$.

From this, we deduce another family of Morse inequalities. Let $\mathcal{M}_{f}^{D}(T)$ be the Morse polynomial

$$
\mathcal{M}_{f}^{D}(T)=\sum_{k}\left|C_{k} \cup N_{k}\right| T^{k}
$$

Let $\mathcal{P}_{(M, \partial M)}^{o r}$ be the relative Poincaré polynomial

$$
\mathcal{P}_{(M, \partial M)}^{o r}(T)=\sum_{k} \operatorname{rank} H^{k}\left(M, \partial M ; \mathbb{Z}^{o r}\right) T^{k}
$$

which is nothing but the symmetric polynomial of $\mathcal{P}_{M}(T)$ :

$$
\mathcal{P}_{(M, \partial M)}^{o r}(T)=T^{n} \mathcal{P}_{M}(1 / T) \text {. }
$$

Corollary B. We have $\mathcal{M}_{f}^{D}(T)-\mathcal{P}_{(M, \partial M)}^{o r}(T)=(1+T) Q^{D}(T)$, where $Q^{D}(T)$ is a polynomial with non-negative coefficients.

Of course, corollaries A and B, which are a direct consequence of theorems A and B respectively, are due to Chang \& Liu [5].

I am very indebted to Francis Nier for encouraging me to return to this question and for his careful reading of a first version. I also thank Jonathan Bloom and Claude Viterbo for useful information and comments.

\section{Proof of Theorem A}

2.1. An adapted pseudo-gradient. We introduce a pseudo-gradient vector field $X$ for the Morse function $f$ adapted to the boundary in the following sense (in case of closed manifolds it is a reformulation of [14] by K. Meyer; a slightly more restrictive, but still generic, definition is given in [8]). 
1) $X . f<0$ except at the critical points in $\operatorname{int}(M)$ and at the critical points of type $N$ on the boundary;

2) $X$ points inwards along the boundary except near the critical points of type $N$ where it is tangent to $\partial M$;

3) if $p \in \operatorname{int}(M)$ is a critical point, $X$ is hyperbolic at $p$ and the quadratic form $\tilde{q}:=X^{\text {lin }} \cdot d_{p}^{2} f$ is negative definite; here $X^{\text {lin }}$ denotes the linear part of $X$ at $p$ (that is, $\left.X^{\text {lin }}=D X(p)\right)$ and the second derivative $d_{p}^{2} f$ at $p$ is thought of as a quadratic function defined near $p$ which is derived by $X^{\text {lin }}$;

4) let $p \in \partial M$ be a critical point of type $N$; there are coordinates $x=(y, z) \in \mathbb{R}^{n-1} \times \mathbb{R}$ of $M$ near $p$, such that $M=\{z \geq 0\}$ and $f(x)=f(p)+z+q(y)$, where $q$ is a non-degenerate quadratic form; it is required that $X$ is a vector field tangent to the boundary, vanishing and hyperbolic at $p$, such that $X^{l i n} \cdot\left(q(y)+z^{2}\right)$ is negative definite;

5) $X$ is Morse-Smale in the sense that the global unstable manifolds and the local stable manifolds are mutually transverse.

Proposition. Given the Morse function $f$, there exists an adapted pseudo-gradient X.

Proof. Look first at condition 4). The existence of coordinates where $f$ reads as $f(x)=$ $f(p)+z+q(y)$ mainly follows from the Morse lemma with parameters (or local stability of Morse functions), up to the addition of a function depending on $z$ only. If $c(z)$ denotes the critical value of $y \mapsto f(y, z)$, the type $N$ assumption implies $\frac{d c(z)}{d z}(0)>0$, hence the normal form holds after changing $z$ by applying the inverse function theorem. Moreover, an easy calculation shows that, away from $\left.p, X^{\text {lin }} \cdot\left(q(y)+z^{2}\right)\right)<0$ implies $\left.X^{l i n} \cdot(q(y)+z)\right)<0$. Therefore, $X$ is a pseudo-gradient for $f$, that is $X . f<0$ near $p$ (except at $p$ ).

Now the local existence of $X$ is clear and local pseudo-gradients can be glued together by a partition of unity. By construction, $X$ is positively complete. Let $X^{t}$ denote its flow; it is defined on an open set of $M \times \mathbb{R}$ containing $M \times[0,+\infty)$. The global unstable manifold $W^{u}(p)$ of a critical point $p$ of index $k$ is the image of a non-proper embedding of $\mathbb{R}^{k}$ into $M$.

There is also a local stable manifold $W_{\text {loc }}^{s}(p)$ which is diffeomophic to $\mathbb{R}^{n-k}$ when $p \in \operatorname{int}(M)$, or to $\mathbb{R}^{n-k} \cap\{z \geq 0\}$ when $p$ is a type $N$ critical point in the boundary (here $\mathbb{R}^{n-k}$ is a space on which $q(y)+z^{2}$ is positive definite). It is properly embedded when it is truncated to the sub-level set $M^{f(p)+\varepsilon}$. Following Smale [18], condition 5) is generically fulfilled among the vector fields meeting conditions 1)-4).

If $p$ has index $k$ the frontier of $W^{u}(p)$, that is the set of points in the closure which are not in $W^{u}(p)$, is contained in the union of the unstable manifolds of critical points of index less than $k$. An orientation of $W^{u}(p)$ is chosen arbitrarily. Then $W_{l o c}^{s}(p)$ is co-oriented by the orientation of $W^{u}(p)$.

Given a pair of critical points $(p, q)$ respectively of index $k$ and $k-1$, there are only finitely many flow lines (up to translation in time) $X^{t}(x), t \in \mathbb{R}$, such that $X^{t}(x) \rightarrow p$ as $t \rightarrow-\infty$ and $X^{t}(x) \rightarrow q$ as $t \rightarrow+\infty$. Each such line has a sign according to the co-orientation of $W_{l o c}^{s}(q)$ with respect to the orientation of $W^{u}(p)$. Let $m_{p q}$ be the algebraic sum of these signs, summing 
up over all connecting orbits from $p$ to $q$.

2.2. The boundary morphism. We are going to define $\partial: F_{k} \rightarrow F_{k-1}$ à la Witten-Floer (see [20, 17). Let $p \in C_{k} \cup N_{k}$ be a generator. The boundary morphism is defined by:

$$
\partial<p>=\sum_{q} m_{p q}<q>
$$

where $q$ runs in $C_{k-1} \cup N_{k-1}$.

Proposition. We have $\partial \circ \partial=0$.

Proof. Consider $p$, a critical point of index $k$, and $q$, a critical point of index $k-2$. We have to prove that $<\partial \circ \partial(p), q>=0$. In [12] it is given a complete description of the closure of $W^{u}(p)$; only the effect of critical points of index $k-1$ is useful for yielding the following description. In a level set $L:=\{f(x)=f(q)+\varepsilon\}$, we look at the trace $A$ of $W_{l o c}^{s}(q)$, a $(n-k+1)$-dimensional sphere or proper disk, and the trace $B$ of the closure of $W^{u}(p)$. The intersection $A \cap B$ lies in the interior of $L$ since $W^{u}(p)$ lies in $\operatorname{int}(M)$ except near $p$ when $p \in \partial M$. It is made of simple closed curves and arcs having end points in common and whose interior are mutually disjoint. The closed curves and the open arcs correspond to connecting orbits from $p$ to $q$; the end points correspond to broken connecting orbits going through some critical points of index $k-1$. Notice that the sign of $m_{p z} m_{z q}$ does not depend on the chosen orientation of the unstable manifold of $z$, an index $(k-1)$ point connected to $p$ and to $q$. Each open arc in $A \cap B$ is oriented. Then one of his end points is equipped with + , the other with - , and the sum of all these signs is $<\partial \circ \partial(p), q>$. Hence, it is zero.

2.3. Invariance. (This property is not needed in the proof of theorems A and B, but it is interesting in itself). Of course, the complex $\left(F^{N}, \partial\right)$ depends on the Morse function $f$ and on an adapted pseudo-gradient $X$. Given two pairs $\left(f_{0}, X_{0}\right)$ and $\left(f_{1}, X_{1}\right)$ where, for $i=0,1, f_{i}$ is a Morse function and $X_{i}$ is an adapted pseudo-gradient, they are connected by a path of pairs $\left(f_{t}, X_{t}\right), t \in[0,1]$, if we allow us to cross "codimension 1 accidents" which arrive at finitely many times $t_{1}, t_{2}, \ldots t_{\ell}$. When $t$ is distinct of those times, $\left(f_{t}, X_{t}\right)$ is a pair of a Morse function and an adapted pseudo-gradient. The list of the possible accidents is yielded by appropriate transversality theorems. We list below the "codimension 1 accidents" for a function $f$; and those of pseudo-gradient $X$. Here, it is convenient to set $f_{\partial}:=f \mid \partial M$.

f1) $f$ has a degenerate critical point at $p \in \operatorname{int}(M)$ and $\operatorname{rank} d^{2} f(p)=n-1$;

f2) $f_{\partial}$ has a degenerate critical point at $p \in \partial M, \operatorname{rank} d^{2} f_{\partial}(p)=n-2$ and $d f(p) \neq 0$;

f3) $d f(p)$ vanishes at some point $p \in \partial M, d^{2} f(p)$ and $d^{2} f_{\partial}(p)$ are both non-degenerate and have the same index;

f4) $d f(p)$ vanishes at some point $p \in \partial M, d^{2} f(p)$ and $d^{2} f_{\partial}(p)$ are both non-degenerate and index $d^{2} f(p)=$ index $d^{2} f_{\partial}(p)+1$.

When crossing an accident f1), a pair of critical points of consecutive indices is created/cancelled in $\operatorname{int}(M)$. When crossing an accident f2), a pair of critical points of consecutive indices and of 
the same type $N$ or $D$ is created/cancelled for $f_{\partial}$ in $\partial M$. Crossing an accident f3) can be modelled as follows: there are local coordinates near $p, x=(y, z) \in \mathbb{R}^{n-1} \times \mathbb{R}$ where $M=\{z \geq 0\}$ and $f(x)=f(p)+z^{2}+q(y)$ where $q$ is a non-degenerate quadratic form (say of index $k$ ). For crossing the accident one leaves this function fixed and translates $M$ by $(y, z) \mapsto(y, z+t)$, $t \in[-\varepsilon,+\varepsilon]$. For $t=\varepsilon$, we have a point of type $N$ and index $k$ on the boundary; for $t=-\varepsilon$, we have a point of type $D$ on the boundary and a new point of index $k$ in $\operatorname{int}(M)$. The model for crossing f4) is similar but $f(x)=f(p)-z^{2}+q(y)$. When moving from $t=\varepsilon$ to $t=-\varepsilon$, a point of type $D$ and index $k$ on the boundary is replaced by a point of type $N$ and index $k$ on the boundary and a point of index $k+1$ in the interior.

We now list the "codimension 1 accidents" for a pseudo-gradient $X$; they never happen at the same time as a "codimension 1 accident" of the function whose $X$ is a pseudo-gradient.

g1) creating/cancelling a pair of connecting orbits from a point of index $\mathrm{k}$ to a point of index $k-1$ (the considered critical points may lie in $\operatorname{int}(M)$ or in $\partial M$ );

g2) there is an orbit connecting two points of the same index.

Proposition. Let $\left(F_{*}^{i}, \partial^{i}\right)$ be the complex associated to the pair of a Morse function and a pseudo-gradient $\left(f_{i}, X_{i}\right), i=0,1$. Then they are quasi-isomorphic: there is a chain morphism from one to the other inducing an isomorphism in homology.

Proof. According to the preceding discussion, we may assume that there exists a path $\left(f_{t}, X_{t}\right)$ with one accident only. The proposition is proved by examining each accident. When crossing f1), an acyclic complex of rank 2 (that is, $0 \rightarrow \mathbb{Z} \cong \mathbb{Z} \rightarrow 0$ ) is added or removed. The same happens when crossing f2), if the considered points are of type $N$; if they are of type $D$ the complex is unchanged. When crossing f3), the complex remains unchanged. When crossing f4), again an acyclic complex of rank 2 is added or removed. Concerning the accidents of $X$, there are those which are encountered in the analogous discussion for closed manifolds (see [12]). In each case we get the desired quasi-isomorphism.

\subsection{The homology of $\left(F_{*}^{N}, \partial\right)$.}

Proposition. The homology of $\left(F_{*}^{N}, \partial\right)$ is isomorphic to $H_{*}(M ; \mathbb{Z})$.

Proof. According to the next lemma, it is allowed to assume that there are no critical points of type $N$. Let us achieve the proof under this assumption. In that case the complex does not "see" the boundary since all connecting orbits lie in int $(M)$. Even, the global unstable manifolds lie in the interior of $M$. So, we can deal with such a function as on a closed manifold. For instance, an adapted pseudo-gradient being chosen, it is possible to reorder the critical values so that the function becomes self-indexing (the critical value of a critical point is its index). Indeed, we recall the following fact in Morse theory (see Cerf [4], II 2.3).

Let $(f, X)$ be a Morse function and an adapted pseudo-gradient. Let $p$ and $q$ two critical points with $f(p)>f(q)$. Assume that the open interval $(f(q), f(p))$ contains no critical value and that there are no connecting orbits from $p$ to $q$. Then there exists a path of 
Morse functions $f_{t}, t \in[0,1]$, with $f_{0}=f, f_{1}(p)<f_{1}(q)=f(q)$ and $X$ is a pseudogradient for every $f_{t}$.

Once $f$ is self-indexing, $M$ has the homotopy type of a CW-complex, with one cell of dimension $k$ for each critical point of index $k$ (see Milnor [17, theorem 7.4, p. 90). In that case, our Morse chain complex is exactly the so-called cellular chain complex. By the cellular homology theorem (see Milnor [16], theorem A.4 p. 263), its homology is the homology of $M$. So the proof will be finished after the following lemma.

Lemma. Let $(f, X)$ be a pair of a Morse function on $M$ and an adapted pseudo-gradient. Let $p$ be a critical point of type $N$ in $\partial M$ and $U$ be a neighborhood of $p$. Then there exist a path of functions $f_{t}$ and an isotopy $\varphi_{t}$ of embeddings $M \rightarrow M, t \in[0,1]$, with $f_{0}=f, \varphi_{0}=I d$, satisfying the following properties :

1) the support of both $f_{t}-f$ and $\varphi_{t}$ is contained in $U$;

2) $f_{t}$ is Morse when $t \neq 1 / 2$;

3) $f_{1}$ has one critical point $p_{1}$ in $U \cap \operatorname{int}(M)$;

4) $f_{1} \mid \partial M$ has one critical point in $U \cap \partial M$ and it is of type $D$;

5) $\varphi_{1}\left(W^{u}(p)\right)$ is the unstable manifold of $p_{1}$ for an adapted pseudo-gradient $X_{1}$ of $f_{1}$, whose other invariant manifolds are those of $X$. In particular, the Morse complexes associated to $X$ and $X_{1}$ are the same.

Of course the accident at time $t=1 / 2$ is f3) in list 2.3.

Proof. We start with a model for $f$ in coordinates $x=(y, z) \in \mathbb{R}^{n-1} \times \mathbb{R}$ on a small open neighborhood $U$ of the critical point $p$ : $f(x)=f(p)+z+q(y)$, where $q(y)$ is a non-degenerate quadratic form and $M \cap U \subset\{z \geq 0\}$. We consider $f_{1 / 2}$ defined on $U$ by $f_{1 / 2}=f(p)+q(y)+z^{2}$. Let $\rho:[0, \delta] \rightarrow[0,1]$ be a smooth bump function: $\rho(s)=1$ for $s$ close to 0 and $\rho(s)=0$ for $s$ close to $\delta$; here $\delta$ is small enough so that the support of $(y, z) \mapsto(\rho(\|y\|), \rho(z))$ is a compact set in $U$. Without loss of generality, we may assume that the support of $\rho$ contains in its interior the connected domain of $\partial M$ along which $X$ is tangent to the boundary.

For $\varepsilon>0$ small enough, we look at the restriction of $f_{1 / 2}$ to $\{z \geq \varepsilon \rho(\|y\|)\}$. It is conjugate to $f \mid M \cap U$ because both functions have no critical points in $U$ and have restrictions to $\partial M \cap U$ which are Morse with one critical point of the same index. Let $M_{0}$ be the closure of $M \backslash\{0 \leq z \leq \varepsilon \rho(\|y\|)\}$ and $\psi: M_{0} \rightarrow M$ be a diffeomorphism such that both functions $f_{0}:=\psi^{*} f$ and $f_{1 / 2}$ have the same germ along $\{z=\varepsilon \rho(\|y\|)\}$. For $t \in[0,1]$, introduce the manifold $M_{t}$ and its function $f_{t}$ obtained from $\left(M_{0}, f_{0}\right)$ by gluing $\{\varepsilon(1-2 t) \rho(\|y\|) \leq z \leq \varepsilon \rho(\|y\|)\}$ endowed with $f_{1 / 2}$. Up to a diffeomorphism $\psi_{t}: M_{t} \rightarrow M$, this path $f_{t}$ is the desired one. 


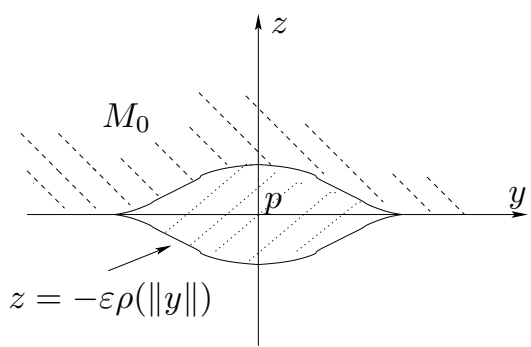

$M_{1}=M_{0} \cup$ the lens

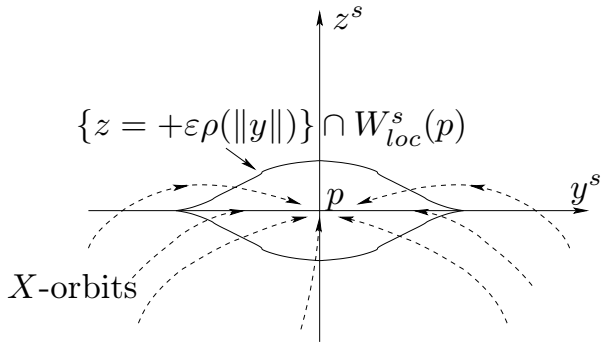

$\left(y^{s}, z^{s}\right)$ stands for coordinates of $W^{s}(p)$

More precisely, the isotopy $\psi_{t}, t \in[0,1]$, can be chosen so that $\psi_{1 / 2}=I d$. Recall that $X$ is a pseudo-gradient in $U$ for both functions $f$ and $f_{1 / 2}$ (see 2.1). At $t=1 / 2$, the deformation of functions is stopped and a small isotopy $\chi_{t}$, is applied for pushing $W^{u}(p) \backslash\{p\}$ into $\operatorname{int}(M)$; it is chosen to be supported in $\operatorname{int}\left(M_{1}\right)$ and to leave $W_{l o c}^{s}(p)$ fixed. As this isotopy is $C^{\infty}$ small, $X_{1}:=\chi_{1 *} X$ is still a pseudo-gradient of $f_{1 / 2}$ and $\chi_{1}\left(W^{u}(p)\right)$ is its unstable manifold of $p$.

Moreover, $X_{1}$ points toward $\operatorname{int}(M)$ along $U \cap\{z=0\}$ except along $W_{l o c}^{s}(p)$. If $\eta>0$ is small, $X_{1}$ points inwards along $\partial M_{1 / 2+\eta} \cap U$. Therefore, if the path of deformation $\left(M_{t}, f_{t}\right)$ is stopped at time $t=1 / 2+\eta$ we already get the desired conclusion.

Remark. (C. Viterbo) Instead of deforming $(f, X)$ as in the preceding lemma, one could invoke Conley's theory of isolating blocks [6] which in our setting states as follows (compare [7] section 3 for a similar situation, but our flow is not negatively complete).

There exists a finite filtration $M_{-1}=\emptyset \subset M_{0} \subset \ldots \subset M_{k} \ldots \subset M_{n}=M$ which is positively invariant by the flow $X^{t}$ and such that $M_{k} \backslash M_{k-1}$ contains all critical points in $C_{k} \cup N_{k}$ and every positive orbit in $M_{k}$ which does not go to a critical point enters $M_{k-1}$ without getting out of.

Our $F_{k}$ can be identified to $H_{k}\left(M_{k}, M_{k-1} ; \mathbb{Z}\right)$ and our $\partial$ is nothing but the connecting morphism $H_{k}\left(M_{k}, M_{k-1} ; \mathbb{Z}\right) \rightarrow H_{k-1}\left(M_{k-1}, M_{k-2} ; \mathbb{Z}\right)$ in the long exact homology sequence of the triple $\left(M_{k}, M_{k-1}, M_{k-2}\right)$ (as in Milnor [16] A.4).

2.5. Complement. We have statements similar to theorem A for every local system of coefficients (or flat bundle with discrete fiber). Such a bundle is given by a representation of the fundamental group $\pi:=\pi_{1}\left(M, x_{0}\right)$ (where $x_{0}$ is a base point) into an Abelian discrete group. For instance, $\mathbb{Z}^{\text {or }}$ is defined by the first Stiefel-Whitney class of $\tau M$, the tangent bundle of $M$ : $w_{1}: \pi \rightarrow \mathbb{Z} / 2$. The complex $F_{*}^{N, \text { or }}$ has the same graded module. But the differential is changed. At the beginning some arc is chosen from the base point to each critical point. Therefore a connecting orbit $\gamma$ from $p$ to $q$ defines a loop $\tilde{\gamma}$ and the contribution of $\gamma$ to $m_{p q}$ is twisted by $w_{1}(\tilde{\gamma})$. So we have a new differential $\partial^{o r}$. The proof that $\partial^{o r} \circ \partial^{o r}=0$ is alike. In that case, theorem A states as the following: The homology of $\left(F_{*}^{N, \text { or }}, \partial^{o r}\right)$ is isomorphic to $H_{*}\left(M ; \mathbb{Z}^{\text {or }}\right)$. New Morse inequalities follow. A good example is given by the compact Möbius band in $\mathbb{R}^{3}$ with a height function. In that case the Betti numbers of $H_{*}\left(M ; \mathbb{Z}^{\text {or }}\right)$ are: $b_{0}=0, b_{1}=0, b_{2}=0$; in relationship to theorem $\mathrm{B}$, we also list the Betti numbers of the relative homology $H_{*}\left(M, \partial M ; \mathbb{Z}^{\text {or }}\right)$ : $b_{0}=0, b_{1}=1, b_{2}=1$. 


\section{Proof of Theorem B And COMplements}

3.1. Upside down. We look at the Morse theory of the Morse function $-f$. The critical points are the same but their indices and types are changed. A critical point in $\operatorname{int}(M)$ of index $k$ for $f$ has index $n-k$ for $-f$. A critical point in $\partial M$ which is of type $\mathrm{D}$ and index $k-1$ for $f_{\partial}:=f \mid \partial M$ (then it belongs to $D_{k}$ ) is of type $N$ and index $n-k$ for $-f_{\partial}$. A critical point in $\partial M$ which is of type $N$ and index $k$ for $f_{\partial}$ is of type $D$ and index $n-k-1$ for $-f_{\partial}$; then it belongs to $N_{n-k}(-f)$.

There is another change to make. Indeed, the vector field $-X$ is not adapted as it points outwards along the boundary except near the critical points of former type $N$. So we appeal another vector field $X^{-}$which is adapted to $-f$. With the data $\left(-f, X^{-}\right)$we can form a Morse complex. But we insist to keep the initial grading, that is: $F_{k}^{D}$ is freely generated by $C_{k} \cup D_{k}$, a set of critical points of index $n-k$ for $-f$. The pseudo-gradient $X^{-}$yields a differential which is of degree +1 in this grading: it is a co-differential and the complex is a cochain complex. According to theorem $\mathrm{A}$ its (co-)homology is isomorphic to $H_{*}(M, \mathbb{Z})$, up to the grading which is changed into the complementary one. Therefore, it is the Poincaré dual of the homology, hence the cohomology with integer coefficients twisted by the orientation. Thus theorem B is proved.

As we did in a variant of theorem A in 2.5, we can twist the codifferential $d$ by the orientation. We get a cochain complex $\left(F_{*}^{D, o r}, d^{o r}\right)$ whose cohomology is isomorphic to $H^{*}(M, \partial M ; \mathbb{Z})$. From this we get another Morse inequalities involving the same Morse polynomial:

$$
\mathcal{M}_{f}^{D}(T)-\mathcal{P}_{(M, \partial M)}(T)=(1+T) Q^{\prime}(T) .
$$

We recall here, that homology and cohomology have the same Poincaré polynomial, as the free factor of $H^{k}($.$) is the dual of the free factor of H_{k}($.$) , as long as the same system of local$ coefficients is used for both.

3.2. Morse inequalities from the double. On the one hand, the set $C_{k}(D f)$ of critical points of index $k$ for the "double" Morse function $D f$ defined on the double $D M$ of $M$ consists of two copies of $C_{k}$, one copy of $N_{k}$ and one copy of $D_{k}$. On the other hand, $H_{k}(D M ; \mathbb{Z}) \cong$ $H_{k}(M ; \mathbb{Z}) \oplus H_{k}(M, \partial M ; \mathbb{Z})$. Indeed, the inclusion $M \hookrightarrow D M$ gives rise to a long exact sequence

$$
\ldots \rightarrow H_{k}(M) \rightarrow H_{k}(D M) \rightarrow H_{k}(M, \partial M) \rightarrow H_{k-1}(M) \rightarrow \ldots ;
$$

but this sequence splits since any relative cycle, glued to its symmetric one, gives a cycle in $D M$. Thus the Morse inequalities for $D f$ is just obtained by adding both Morse inequalities obtained previously, the one for $\mathcal{M}_{f}^{N}-\mathcal{P}_{M}$ and the one for $\mathcal{M}_{f}^{D}-\mathcal{P}_{(M, \partial M)}$ :

$$
\mathcal{M}_{D f}(T)-\mathcal{P}_{D M}(T)=(1+T) Q^{\prime \prime}(T) .
$$

Thus, it is weaker than its summands.

3.3. Relative homology and its pairing with absolute homology. 1 Here we need the following notation: for $p \in C_{k} \cup D_{k}, W^{u}\left(p, X^{-}\right)$will denote the unstable manifold of the pseudogradient vector field $X^{-}$adapted to the function $-f$; it is equipped with an orientation. If we want to think of it as a relative $k$-cochain, it is necessary to endow $p$ with a local orientation

${ }^{1}$ I thank M. Akaho for asking me about the relative homology. 
of $M$. Then, if $\sigma$ is an oriented $k$-simplex tranverse to $W^{u}\left(p, X^{-}\right)$it is allowed to count the intersection points in $\sigma \cap W^{u}\left(p, X^{-}\right)$with signs. It is the way of thinking of the complex $\left(F_{*}^{D}, d\right)$ as a cochain complex with orientation-twisted coefficients calculating $H^{*}\left(M, \partial M ; \mathbb{Z}^{o r}\right)$.

Now, we consider the dual complex $\left(\check{F}_{*}^{D}, \breve{d}\right)$ in which the differential is the transpose of $d$. Then its homology is isomorphic to $H_{*}\left(M, \partial M ; \mathbb{Z}^{o r}\right)$; here we use the universal-coefficient theorem for cohomology ([19], theorem 5.5.3) and the finite generation of $\left(F_{*}^{D}, d\right)$. Geometrically, $\check{d}$ can be described as follows. Let $p \in C_{k} \cup D_{k}$ and $q \in C_{k-1} \cup D_{k-1}$. We have to calculate $\check{m}_{p q}:=<\check{d}(p), q>$. It is the algebraic number of connecting orbits of $X^{-}$from $q$ to $p$ counted with the orientation of $W^{u}\left(q, X^{-}\right)$and the co-orientation of $W_{l o c}^{s}\left(p, X^{-}\right)$.

For making the pairing $H_{k}\left(M, \partial M ; \mathbb{Z}^{\text {or }}\right) \otimes H_{n-k}(M ; \mathbb{Z}) \rightarrow \mathbb{R}$ explicit, we need to ask a general position of $X^{-}$with respect to $X$ : for each pair of integers $\left(k, k^{\prime}\right)$ and for each $p \in C_{k} \cup D_{k}$ and $p^{\prime} \in C_{n-k^{\prime}} \cup N_{n-k^{\prime}}$ the unstable manifold $W^{u}\left(p, X^{-}\right)$is transverse to the unstable manifold $W^{u}\left(p^{\prime}, X\right)$. When $k=k^{\prime}$, it is easy to see that only compact parts of these invariant manifolds could intersect; therefore, there are finitely many intersection points. The sign is calculated by using three data; the orientation of $W^{u}\left(p^{\prime}, X\right)$, the orientation of $W^{u}\left(p, X^{-}\right)$and the local orientation of $M$ at $p$.

Arguing this way, we avoid to identify $\check{F}_{*}^{D}$ to a quotient complex of $F_{*}^{N}$ by a subcomplex isomorphic to the Morse complex of $f \mid \partial M$. Such an identification should require difficult formulas similar to those in 9], section 2.4.

\section{REFERENCES}

[1] M. Akaho, Morse homology and manifolds with boundary, Commun. Contemp. Math. 9 no. 3 (2007), 301334.

[2] M. Braverman, V. Silantyev, Kirwan-Novikov inequalities on a manifold with boundary, Trans. Amer. Math. Soc. 358 (2006), no. 8, 3329-3361.

[3] R. Bott, Lectures on Morse theory, old and new, Bull. Amer. Math. Soc. (N.S.) 7 (1982), no. 2, 331-358.

[4] J. Cerf, La stratification naturelle des espaces de fonctions différentiables réelles et le théorème de la pseudoisotopie, Inst. Hautes Études Sci. Publ. Math. 39 (1970), 5 - 173.

[5] K.C. Chang, J. Liu, A cohomology complex for manifolds with boundary, Top. Methods in Non Linear Analysis 5 (1995), 325 - 340.

[6] C. Conley, Isolated invariant sets and the Morse index, CBMS Regional Conference Ser. in Math. 38, Amer. Math. Soc., 1978.

[7] A. Floer, Witten's complex and infinite dimensinal Morse theory, J. Diff. Geom. 30 (1989), 207-221.

[8] V. Grines, F. Laudenbach, O. Pochinka, Self-indexing energy function for Morse-Smale diffeomorphisms on 3-manifolds, Moscow Mathematical Journal 9 no. 4 (2009), 801-821.

[9] P. Kronheimer, T. Mrowka, Monopoles and three-manifolds, New Math. Monographs 10, Cambridge Univ. Press, 2007.

[10] B. Helffer, F. Nier, Quantitative analysis of metastability in reversible processes via a Witten complex approach: the case with boundary, Mém. Soc. Math. Fr. 105 (2006).

[11] N. Koldan, I. Prokhorenkov, M. Shubin, Semiclassical asymptotics on manifolds with boundary, in: Spectral analysis in geometry and number theory, 239-266, Contemp. Math., 484, Amer. Math. Soc., 2009.

[12] F. Laudenbach, On the Thom-Smale complex, Astérisque 205 (1992), 219 - 233.

[13] D. Le Peutrec, Small eigenvalues of the Neumann realization of the semiclassical Witten Laplacian, to appear in Ann. Fac. Sci. Toulouse Math.

[14] K. Meyer, Energy functions for Morse-Smale systems, Amer. J. Math. 90 (1968), 1031-1040. 
[15] J. Milnor, Morse theory, Annals of Math. Studies 51, Princeton Univ. Press, 1963.

[16] J. Milnor, J. Stasheff, Characteristic classes, Annals of Math. Studies 76, Princeton Univ. Press, 1974.

[17] J. Milnor, Lectures on the h-cobordism theorem, Princeton Univ. Press, 1965.

[18] S. Smale, On gradient dynamicalsystems, Annals of Math. 74 (1961), 199-206.

[19] E. Spanier, Algebraic topology, McGraw-Hill, 1966.

[20] E. Witten Supersymmetry and Morse theory, J. Diff. Geom. 17 (1982), 661-692.

Laboratoire de mathématiques Jean Leray, UMR 6629 du CNRS, Faculté des Sciences et Techniques, Université de Nantes, 2, rue de la Houssinière, F-44322 Nantes cedex 3, France. E-mail address: francois.laudenbach@univ-nantes.fr 